\title{
Energy Efficient Strategies with Mobile Sink for WSNs: A Survey
}

\author{
Feng Ruan ${ }^{1}$, Zhiyong Fan $^{1}$, Yiguang Gong ${ }^{1}$, Jianmin Hou $^{1}$, Ping Mei ${ }^{1}$ \\ and $\mathrm{Tao} \mathrm{Li}^{12}$ \\ 1 \\ School of Information \& Control, Nanjing University of Information Science \& \\ Technology, Nanjing, China \\ ${ }^{2}$ School of Electronic \& Information Engineering, Nanjing University of \\ Information Science \& Technology, Nanjing, China
}

\begin{abstract}
Sink mobility has been widely used to reduce the energy consumption of sensor nodes, which can prolong the lifetime of nodes in wireless sensor networks (WSNs). Researchers found that sink mobility can improve the stability, throughput and lifetime of the very large-scale WSNs. However, sink mobility also causes issues such as data latency and communication overhead. With these properties sink mobility remains more research efforts under different requirements. In this paper we present a survey of energy efficient strategies in WSNs with mobile sink. Firstly, we investigate the existing issues in WSNs. Then we focus on the problem of energy efficient data gathering strategy with mobile sink and present some existing solutions. Finally, we conclude this paper with some potential extensions which need to be further explored.
\end{abstract}

Keywords: Wireless sensor networks, sink mobility, energy efficiency, data gathering.

\section{Introduction}

A wireless sensor network (WSN) consists of two units: a great deal of tiny sensor nodes and a remote sink node (or base station). Sensor nodes, which are powered by batteries, are deployed to gathering data in un-attended or dangerous environments. This makes it impossible to replace the battery of nodes. So the most important issue for WSNs is minimizing the energy consumption of sensor nodes.

In WSNs with static sink, energy of sensor nodes in the vicinity of sink deplete quicker than other nodes because of the concentration of data traffic towards the sink, which give rise to the hotspot problem [1]. Sensor nodes near the sink will die quickly and the sink will be isolated, the data gathered across the network will no longer be transmitted to the sink. Therefore, sink mobility are proposed to tackle this issue [2-4]. With the sink moves, the hotspot area will change. This can help uniform the energy consumption of sensor nodes and prolong the lifetime of the whole network. However, sink mobility also contributes to issues such as data latency and communication overhead. Thus, the defects of sink mobility should be taken into consideration before using it.

WSNs can be used in a variety of daily life such as medical healthcare [5]. Sensor nodes are designed to be easy to carry on human bodies (such as a watch) so that the healthcare professionals can gather data for further analysis. Also, it can be used to monitor battlefield [6], once a sensor node detects an event (a sound), it will transmit data to the sink or base station. Moreover, a WSN can be used for forest fire detection systems [7], nodes report the temperature and humidity to the sink carried by forest ranger or a motorized vehicle periodically.

The main contribution of this paper is to provide an overall survey on the energy efficiency routing protocols for WSNs with mobile sink. We address on the strategies these protocols used to transmit data, considering the energy they consume and how they 
achieve this. Moreover, we discuss the benefits and defects of each protocol with some metrics (data latency, stability and power consumption). Other surveys on the mobile sink routing in WSNs exists in the literature [3-5, 8]. Our survey focus on energy efficient strategies with mobile sink in WSNs, we aim to introduce a more detailed research on this project.

The rest of this paper is organized as follows: Section 2 gives an overview of movement pattern of mobile sink in WSNs and some design issues. Section 3 describes the existing energy efficient routing strategies in WSNs with mobile sink and address on the benefits and defects of them. Section 4 gives an open topic to discuss the aspects that needs more research effort. Finally, Section 5 concludes the paper.

\section{Movement Patterns based on Mobile Sink}

Recently, many researches have been conducted on the issue of prolong the lifetime of a WSN through the management of mobile sink $[2,3]$. We can classify sink mobility into three categories: random mobility, predictable mobility and controllable mobility. Table 1 shows the characteristics, issues and examples of each category.

Table 1. Classification of Sink Mobility Approaches

\begin{tabular}{|c|c|c|c|}
\hline Approaches & Characteristics & Issues & Examples \\
\hline $\begin{array}{c}\text { Random } \\
\text { Mobility }\end{array}$ & Sinks randomly move & $\begin{array}{c}\text { data packet drop, } \\
\text { data latency }\end{array}$ & $\begin{array}{c}\text { TTDD[9], } \\
\text { HCDD[10] }\end{array}$ \\
\hline $\begin{array}{c}\text { Predictable } \\
\text { Mobility }\end{array}$ & $\begin{array}{c}\text { Sinks moving along } \\
\text { predefined trajectory }\end{array}$ & data latency & VGDRA[14] \\
\hline $\begin{array}{c}\text { Controllable } \\
\text { Mobility }\end{array}$ & $\begin{array}{c}\text { Sinks move to the } \\
\text { interest area }\end{array}$ & $\begin{array}{c}\text { Motion control, } \\
\text { data latency }\end{array}$ & BRH-MDC[12] \\
\hline
\end{tabular}

For the random mobility approach, the structure of the network is easy to construct. Sink randomly move along the network and gathering data from the sensor nodes among the communication range. Also, this approach barely bring communication overhead to nodes since the sink directly communicate with them. The defects of this approach are data latency and low reliability. Sensor nodes have to wait for sink to disseminate data, which give rise to large delays. Moreover, the buffer of the nodes might overflow and the data package might be dropped.

For the predictable mobility approach, sinks move on pre-defined trajectories, e.g., freeways, railways, etc. Nodes can calculate the location of the sink and choose a suitable route to transmit data. This approach can improve the storage overheads and provide high reliability. However, the network structure is inflexible. Once the network topology changes, the performance of this approach will decrease.

For the controllable mobility approach, sinks move to the interest areas, e.g., the traffic load of the network and quality of service. It provides a flexible moving strategy which can maintain a balance between prolonging network lifetime and decreasing data latency. However, this approach involves both motion control and data routing issues, it requires extra hardware and calculation resources to realize this function.

There are two main metrics to measure an efficient mobile sink routing protocol: minimizing data latency of data delivery and limiting energy consumption of nodes. On one hand, sink mobility may give rise to data latency and contribute to sensor nodes' data buffer overflow, which will give rise to data package loss and decrease the reliability of the WSN. On the other hand, sink mobility can reduce multi hop routing, which can decrease the communication overhead and reduce energy consumption of the sensor nodes. Considering the different requirements of WSNs is necessary before design a mobile sink routing protocol. 
Also, there are some issues need to be considered before designing energy efficient routing protocols with mobile sink:

- Motion control. This issue involves both sink speed control and moving trajectory design. For predictable mobility, speed control should be considered. For uncontrolled mobility, both speed control and trajectory design is under consideration. But for random mobility, there is no such issue since the sink is not under control.

- Data latency. A sensor node is far from a sink or the network traffic overload will give rise to latency. Also, the time for sensor nodes locate the position of a sink may cause latency. This is an important issue especially for a real-time application.

- Reliability. Minimizing the data packet loss can increase the reliability of the network. The data buffer overflow of sensor nodes or the sudden increase of traffic load of the network will lead to packets loss. A good routing protocol must decrease this loss. One possible solution is using data fusion to reduce data flow of the network; another solution is optimizing the motion control of sink. It is a significant measure for an efficient WSN

\section{Data Gathering Strategies of WSNs Based on Mobile Sink}

In WSNs, location of the sink is usually unpredictable, and can lead to network topology changes unexpectedly. Though there has been a great deal of researches on this topic, providing an up-to-date location of mobile sink with low cost is a hard work. Since tremendous research efforts have been devoted to the development of WSNs, we now divide the data gathering strategies into two parts: delay-torrent routing [13] and delaysensitive routing [9].

In this section, we present a comprehensive review of energy efficient routing protocols of WSNs with mobile sink. We first introduce delay-torrent routing strategy, which is most energy efficiency since it produces less overhead of the network; then we introduce delay-sensitive routing strategy, which produces huge communication overhead for location update and mobility-aware data routing.

\subsection{Delay-torrent Routing}

In delay-torrent routing strategy, data packages are not necessarily needed to be disseminated to the sink in real time and it could be delayed for hours or days. The data packets are stored in buffer (Figure 1) or sent to rendezvous point (RP) (Figure 2), it will be disseminated later when a mobile sink move among the transmit range of sensor nodes.

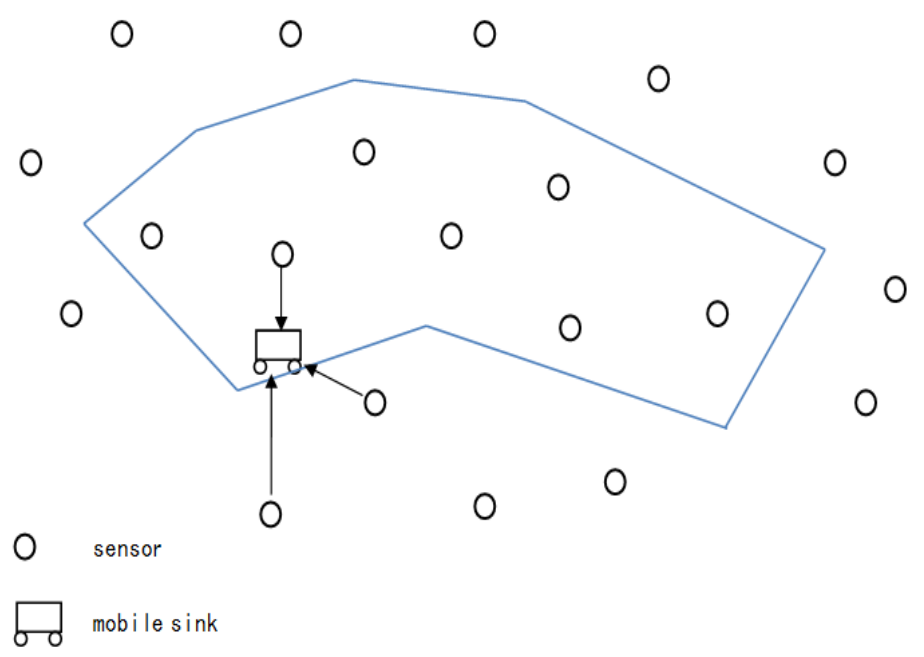

Figure 1. Data Packets Store in Buffer 


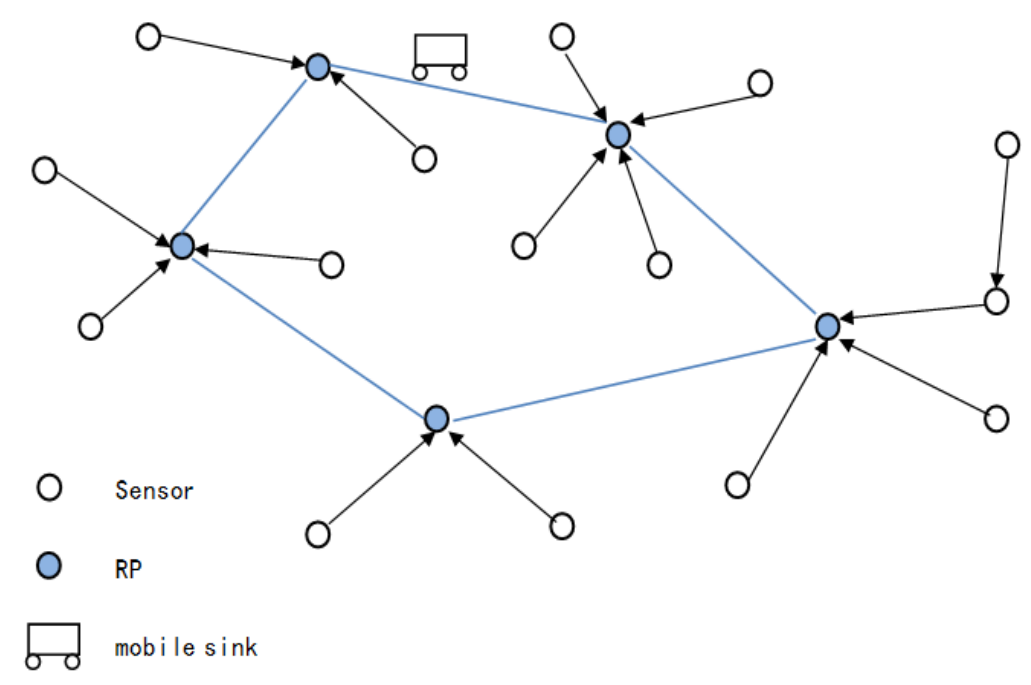

Figure 2. Data Packets Transmit to Rendezvous Point

Below we will introduce some existing delay-torrent routing algorithms in details.

1) Data Mules: Shah et al. present three-tier mule architecture do decrease energy consumption of nodes and reduce infrastructure cost [13]. Figure 3 shows the architecture of the network. MULEs are mobile agents such as people, animals or vehicles (cars, buses). MULE communicate with sensors or an access point (AP) via single hop routing when a MULE moves among the communication range. The upper layer APs connect with outer network.

Advantages: Data MULEs provide a solution for connecting large sparse sensor network. Owing to the single-hop routing, it can decrease the energy consumption of sensor nodes and relief communication overhead of the network. Also, infrastructure cost of the network is low.

Drawbacks: Data latency of this approach is extremely high, which limits the types of applications this solution would be applicable for. Also, if the data buffer of the sensor nodes or MULEs is full, all extra data will be dropped, which can decrease the reliability of the network.

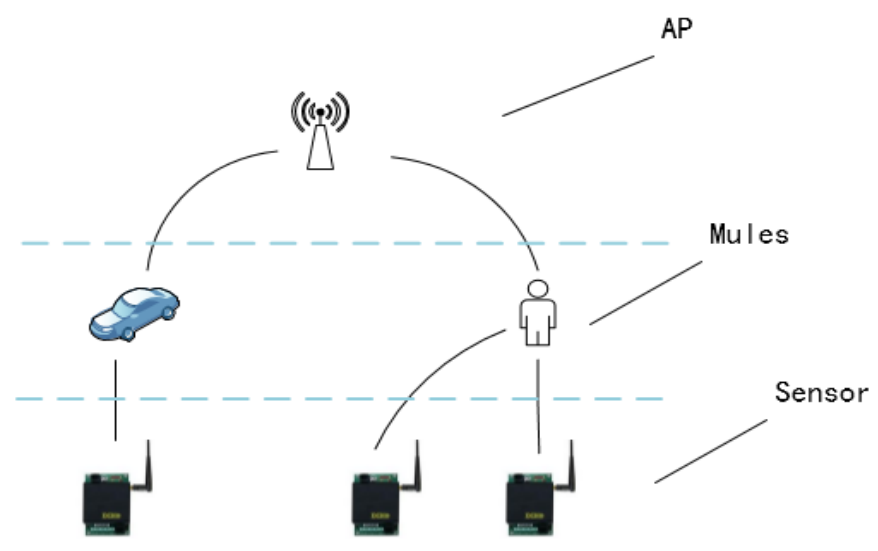

Figure 3. MULEs Three-layer Architecture

2) TTDD: Luo et al. present a Two-Tier Data Dissemination approach [9] to address the multiple, mobile sink problem. When a sensor captures an event, it will become a 
source node that will generate data reports. Then the source node will be chosen as a grid point and the whole network will be divided into grids with a predefined size. It updates forward information of the sensor to the closest grid point.

Advantages: This approach divides the network into girds, and thus avoids flooding the whole network for sink location. It solves the problem of data dissemination problem by utilizing a grid structure, which relief the communication overhead of the network.

Drawbacks: Data routing of this protocol can be optimized due to its flood-based feature. In very large-scale WSNs, the communication overhead could be high. Also, it consumes extra energy because each source node has to build a virtual grid structure to communicate with mobile sink

3) DQM: Data Quality Maximization (DQM) [19] is a backbone-based routing protocol. It consists of three tiers: common sensors at bottom tier; gateways (the sensors that are within the transmission range of the mobile sink) at middle tier; mobile sink at upper tier. Besides, gateways aggregate incoming data and transmit data with sink in single-hop routing.

Advantages: This approach eliminates energy consumption through single-hop routing between gateways and mobile sink. Also, data aggregation can reduce communication overhead.

Drawbacks: DQM reduces energy consumption at the cost of long data latency. Besides, many-to-one communication consumes the energy of gateways quicker than common nodes, which give rise to hotspot problem.

4) Subflow-based and Queue-based models: In [20], Yun and Xia proposed a framework to improve the network lifetime; it reduces energy consumption at the cost of data latency. Mobile sink stops at a stop and receive data from sensors within this location. This framework is divided into two models through the data buffer strategy: subflowbased model and queue-based model.

Advantages: This framework effectively prolong the lifetime of sensor nodes. It provides two different models that can be conducted on different scenarios.

Drawbacks: It neglects the traveling time of mobile sink between each stop. Also, trajectory design of sink mobility is a big issue.

\subsection{Delay-sensitive Routing}

In delay-sensitive routing strategy, data packages should be transmitted to mobile sink in time via multi-hop routing. Frequent routing update is needed to transmit data packets to mobile sink in time (Figure 4). For this strategy, the communication overhead of the whole network is extremely high, which consume much energy of sensor nodes. It can also give rise to data buffer overflow and lead to data packets loss. Minimizing the communication overhead is a key issue to design such protocols. 


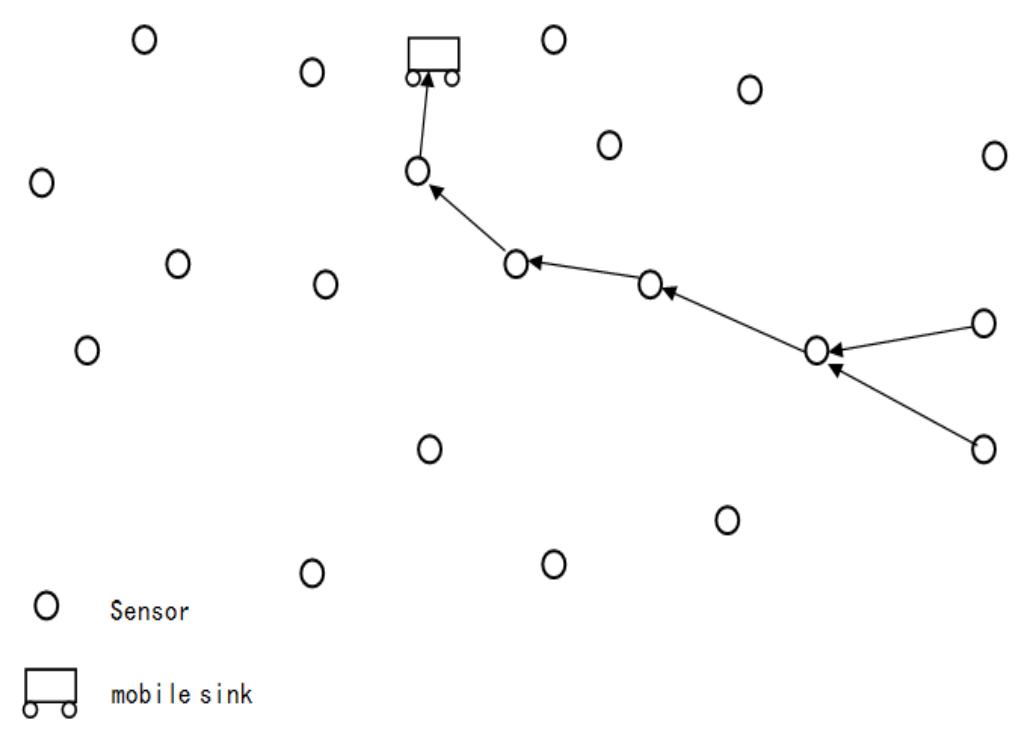

Figure 4. Data Packets Transmit From Source Node to Mobile Sink

Below we will introduce some existing delay-sensitive routing algorithms in details.

1) Joint Sink Mobility and Routing: Luo and Hubaux in [2] analyzed the network energy consumption when mobile sink moves in different trajectory. They researched in theoretical analysis and mathematic simulation, and then found that when sensors are deployed in circle, the most energy efficient moving trajectory for mobile sink is to move along the edge of the network. Also, they propose algorithm joint sink mobility and routing, they proved that a mobile sink is better than a static sink.

Advantages: They proposed a theoretical proof on sink mobility can improve network performance.

Drawbacks: This work focuses on theoretically analysis. However, in real-world WSN, constraint to the landscape, deployment of sensors is no dense. Also, in large scale WSN, this approach will give rise to data latency.

2) GMRE and MILP: Greedy Maximum Residual Energy (GMRE) [16] and Mixed Integer Linear Programming (MILP) [17] are both motion control strategy to transform single sink. In GMRE, sink will move to the high residual energy area to balance network energy consumption. In MILP, it provides a centralized solution. It needs to have a global view of network topology, communication costs, etc.

Advantages: Both of them can improve the performance of the network.

Drawbacks: For GMRE, updating residual energy of the whole network will bring great communication overhead, which will decrease the performance of network. For MILP, centralized management is hard to conduct and it requires large calculation. Also, it faces overhead problems.

3) VGDRA: Khan et al. present A Virtual Grid-Based Dynamic Routes Adjustment Scheme [14] to balance energy consumption and data latency with one mobile sink. The network was divided into $\mathrm{K}$ uniform sized cells; each cell has a fixed cell-header, which is used for collect data from member nodes and transmits data to mobile sink. Then define the cell-headers, a virtual backbone network is constructed to connect each cell-header. Mobile sink only communicate with the closest cell-header, as sink moves, it communicate with different cell-headers. Figure 5 shows the network structure.

Advantages: By using proposed rules, only limited cell-headers need to reconstruct routes. This approach can effectively reduce communication costs, and thus prolong the lifetime of WSN. Also, multi-hop routing can relief the data latency.

Drawbacks: Cell heads will die quickly because of communication overhead, and reduce the life time of network. Also, in sparse network, this approach is not that efficient. 


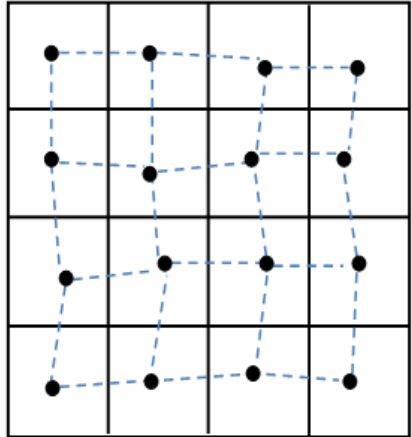

(a)

- Cell-header

-..- Communication Path

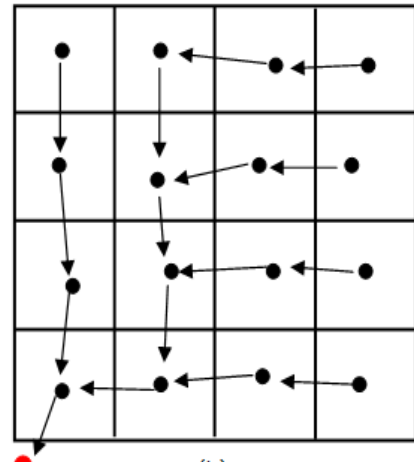

(b)

- Mobile Sink

$\longleftarrow$ Data Delivery Path

$\longleftarrow$ Update Data Delivery Path

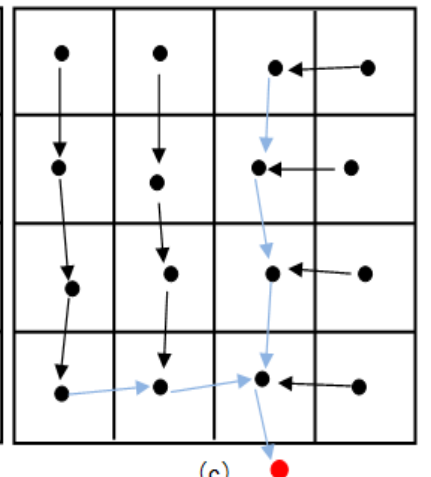

(c)

Figure 5. Network Structure of VGDRA

4) EECLA: Nagamalar et al. propose an Energy Efficient Cluster based Approach for Data Collection with Multiple Mobile Sink [15], it is an improved approach based on VGDRA. The key idea of this approach is optimizing routing scheme by using multiple mobile sink. Compared to VGDRA, this scheme has several improvements: Node which is close to grid center and has high residual energy will be chosen as cluster head. Also, the network is divided into two parts, each sink communicate with one part. Figure 6 shows the network structure.

Advantages: Using residual energy as a metric can prolong the lifetime of sensor nodes and improve the performance of WSN. Also, the multi sinks can reduce the data latency problem.

Drawbacks: Moving speed of mobile sink is not under consideration in this approach. Moreover, the capability of the network is poor. When the number of nodes increases, the network structure will be reconstructed.

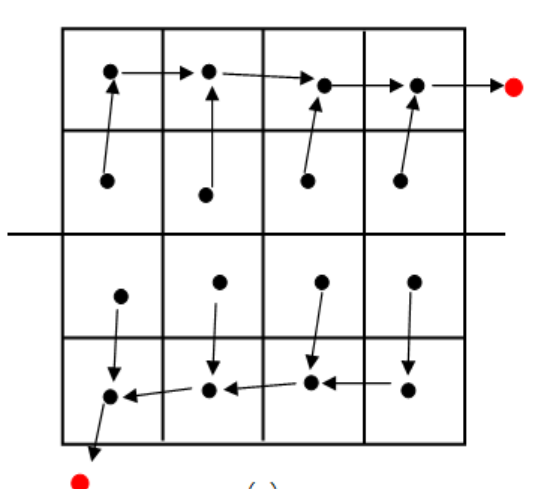

(a)

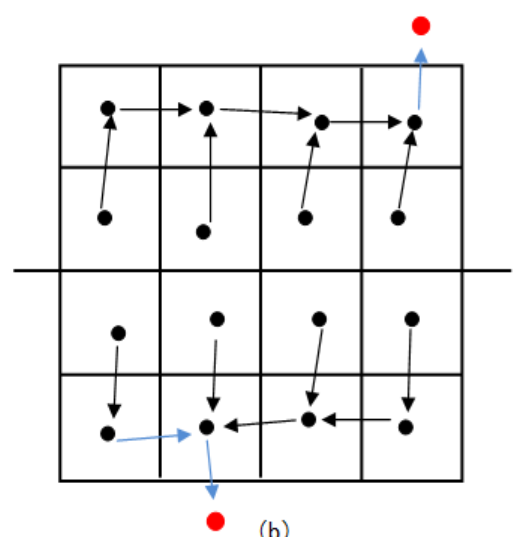

(b)

- Mobile Sink

- Cluster Head

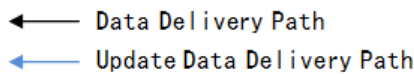

Figure 6. Network Structure of EECLA 


\section{Open Topics}

Although great research effort has been made on sink mobility, there are still many aspects need to be further explored.

1) Delay-torrent and delay-sensitive network. In most researches, we assume the network as a delay-torrent or delay-sensitive network. But for some scenarios, the network will produce both delay-torrent data packets and real-time packets. For example, in forest fire monitoring, sensor nodes report monitored data periodically, which are delay-torrent. Once a sensor node detects violent changes of temperature, it will send an alarm package which needs to be transmitted to sink immediately. So, QoS routing should be taken into consideration in future research.

2) Energy consumption of data processing. Most strategies assume that the energy consumption of sending and receiving data are far more than that in sensing and processing data. In real-word applications, when the data transmission period is relatively long, the energy consumption of sensing and processing data may cost more than that in data transmission. In this case, a more accurate mobility model is needed in designing an energy efficient routing protocol.

3) Sink mobility speed. In random mobility models, sink moves randomly and sink speed is not under control. But in other mobility models, implying speed control seems to be feasible in achieving a balance between energy efficient and data latency. When a sink moves slowly, the routing topology changes slowly and requires less location update packets, however, this approach will increase data latency since it takes more time for mobile sink to travel between sensor nodes. On the contrary, fast moving speed for mobile sinks will give rise to frequent location update, which may cause communication overhead.

4) QoS routing protocols. In some applications, reliable delivery of data is extremely important. For example, in healthcare, doctors need the real-time information of patients; data latency and data package loss are not allowed. Meanwhile, in this application, bandwidth should be guaranteed. However, this application will consume more energy of sensors and decrease network lifetime. More research efforts are need in this aspect.

5) Energy of batteries. In WSNs, the energy of sensors is limited and their batteries cannot be replaced. This is why we research energy efficiency in WSNs. But for outdoor applications, there are some extra alternative energy supplies. For example, green energy resources like solar and wind are good choices for sensors. But this may increase the cost of WSN, cheaper green energy can be considered in future research.

\section{Conclusions}

In this paper, we present a review of energy efficient strategies with mobile sink in WSNs. We classify the movement patterns into three categories: Random Mobility, Predictable Mobility and controllable Mobility. Each of them has its advantages and weaknesses. Then we investigate data gathering approaches: delay-torrent routing and delay-sensitive routing. The former approach focuses on energy efficient the latter concentrates on decrease data latency. Through the existing works, we can see that sink mobility can enhance the network performance.

Although many researchers have concentrated on energy efficient strategies and achieved much progress, there are still many aspects need to be further explored. For instance, delay-torrent and delay-sensitive network as well as energy consumption in data processing still need more research effort. Also, sink speed and QoS routing protocols are promising research aspects. Last, designing sustainable energy for sensors could be an alternative solution to prolong network lifetime. 


\section{Acknowledgements}

This paper is a revised and expanded version of a paper entitled "Energy Efficient Strategies in Wireless Sensor Networks with Mobile Sink" presented at CIA 2016 Philippines, May 19-21. This work was supported by the National Natural Science Foundation of China (71503136, 61304089). It was also supported by the China Special Fund for Meteorological Research in the Public Interest under grant GYHY201306070, and by the Priority Academic Program Development of Jiangsu Higher Education Institutions.

\section{References}

[1] J. Lian, K. Naik and G. B. Agnew, "Data capacity improvement of wireless sensor networks using nonuniform sensor distribution”, International Journal of Distributed Sensor Networks, vol. 2, no. 2, (2006), pp. 121-145.

[2] M. Di Francesco, S. K. Das and G. Anastasi, "Data collection in wireless sensor networks with mobile elements: A survey", ACM Transactions on Sensor Networks (TOSN), vol. 8, no. 1, (2011), p. 7.

[3] Y. Gu, F. Ren, Y. Ji, and J. Li, "The evolution of sink mobility management in wireless sensor networks: A survey", (2015).

[4] H. Alemdar and C. Ersoy, "Wireless sensor networks for healthcare: A survey", Computer Networks, vol. 54, no. 15, (2010), pp. 2688-2710.

[5] I. Bekmezci and F. Alagöz, "Energy efficient, delay sensitive, fault tolerant wireless sensor network for military monitoring", International Journal of Distributed Sensor Networks, vol. 5, no. 6, (2009), pp. 729-747.

[6] C. Tunca, S. Isik, M. Y. Donmez and C. Ersoy, "Distributed mobile sink routing for wireless sensor networks: a survey", Communications Surveys \& Tutorials, IEEE, vol. 16, no. 2, (2014), pp. 877-897.

[7] H. Luo, F. Ye, J. Cheng, S. Lu and L. Zhang, "TTDD: Two-tier data dissemination in large-scale wireless sensor networks", Wireless networks, vol. 11, no. 1-2, (2005), pp. 161-175.

[8] U. Lee, B. Zhou, M. Gerla, E. Magistretti, P. Bellavista and A. Corradi, "Mobeyes: smart mobs for urban monitoring with a vehicular sensor network", Wireless Communications, IEEE, vol. 13, no. 5, (2006), pp. 52-57.

[9] M. Zhao and Y. Yang, "Bounded relay hop mobile data gathering in wireless sensor networks", Computers, IEEE Transactions, vol. 61, no. 2, (2012), pp. 265-277.

[10] R. Shah, S. Roy, S. Jain and W. Brunette, "Data MULEs: Modeling a three-tier architecture for sparse sensor networks", IEEE Snpa Workshop, vol. 1, no. 1, (2003), pp. 30-41.

[11] A. W. Khan, A. H. Abdullah, M. A. Razzaque and J. I. Bangash, "VGDRA: a virtual grid-based dynamic routes adjustment scheme for mobile sink-based wireless sensor networks", Sensors Journal, IEEE, vol. 15, no. 1, (2015), pp. 526-534.

[12] S. Basagni, A. Carosi, E. Melachrinoudis, C. Petrioli and Z. M. Wang, "Controlled sink mobility for prolonging wireless sensor networks lifetime", Wireless Networks, vol. 14, no. 6, (2008), pp. 831-858.

[13] M. Zhao and Y. Yang, "Bounded relay hop mobile data gathering in wireless sensor networks", Computers, IEEE Transactions, vol. 61, no. 2, (2012), pp. 265-277.

[14] Y. Yun and Y. Xia, "Maximizing the lifetime of wireless sensor networks with mobile sink in delaytolerant applications", Mobile Computing, IEEE Transactions, vol. 9, no. 9, (2010), pp. 1308-1318.

[15] J. Luo and J. P. Hubaux, "Joint mobility and routing for lifetime elongation in wireless sensor networks", INFOCOM 2005. 24th annual joint conference of the IEEE computer and communications societies, Proceedings IEEE, vol. 3, (2005), pp. 1735-1746.

[16] L. Yu, N. Wang and X. Meng, "Real-time forest fire detection with wireless sensor networks", Wireless Communications, Networking and Mobile Computing, 2005, Proceedings, 2005 International Conference, vol. 2, (2005), pp. 1214-1217.

[17] C. J. Lin, P. L. Chou and C. F. Chou, "HCDD: hierarchical cluster-based data dissemination in wireless sensor networks with mobile sink", Proceedings of the 2006 international conference on Wireless communications and mobile computing, ACM, (2006), pp. 1189-1194.

[18] T. Nagamalar and T. R. Rangaswamy, "Energy efficient cluster based approach for data collection in wireless sensor networks with multiple mobile sink", Industrial Instrumentation and Control (ICIC), 2015 International Conference, (2015), pp. 348-353.

[19] S. Basagni, A. Carosi, E. Melachrinoudis, C. Petrioli and Z. M. Wang, "A new MILP formulation and distributed protocols for wireless sensor networks lifetime maximization”, Communications, 2006. ICC'06, IEEE International Conference, vol. 8, (2006), pp. 3517-3524.

[20] X. Xu, W. Liang and T. Wark, "Data quality maximization in sensor networks with a mobile sink", Distributed Computing in Sensor Systems and Workshops (DCOSS), 2011 International Conference, (2011), pp. 1-8. 

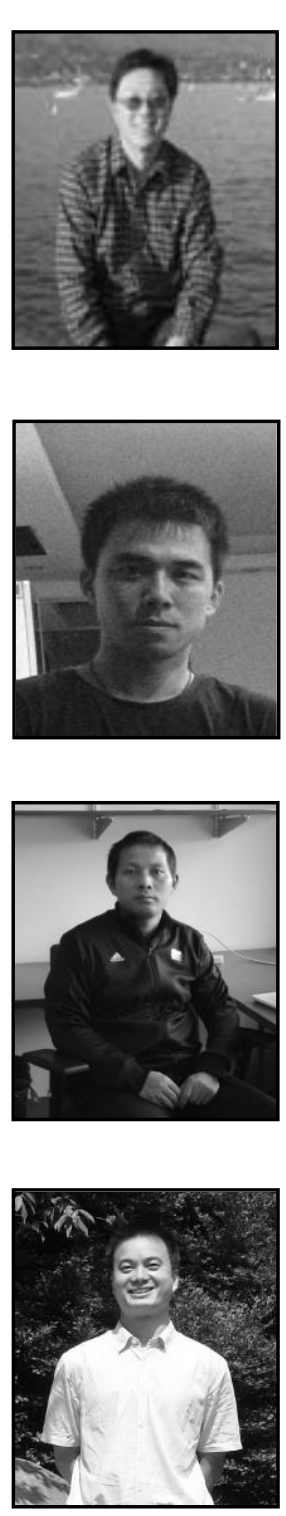

\section{Authors}

Feng Ruan, he is a lecturer at Nanjing University of Information Scicence\&Technology. He received the B.S. and M.S. degree in the Nanjing University of Information Scicence\&Technology.Now he is PHD student in Computer Science Department of Nanjing University of Information Scicence\&Technology. His research interests mainly includecomplex system, computer network, routing protocol and algorithm design, data minining,cloud computing.

Zhiyong Fan, he received MSc from Nanjing University of Information Science and Technology (Nuist) in 2007, China. He is a lecturer in the School of Information and Control Engineering at Nuist, China. Now, he is a PhD student in Nanjing University of Science and Technology, China. His current research interests include medical imaging, image processing and pattern recognition.

Yiguang Gong, he is a lecturer at Nanjing University of Information Science \& Technology. He received the B.S. and M.S. degree in the China University of Geosciences, and PHD in Nanjing University of Aeronautics and Astronautics. His research interests mainly include web information extraction, machine learning, embedded systems, .Wireless Sensor Networks.

Tao Li, he is an associate Professor at School of Electronic \& Information Engineering, Nanjing University of Information Science \& Technology. He received the B.S. and M.S. degree in Nanjing University of Information Science \& Technology, and PHD in Nanjing University of Aeronautics and Astronautics. His research interests mainly include computer network, big data, data mining, and cloud computing. 